\title{
Analysis and Research on TTNT Data Link
}

\author{
Wenqing Zheng ${ }^{*}$, a), $\mathrm{Hu} \mathrm{Jin}^{*}{ }^{*, \mathrm{~b})}$,Yifan Liu $\left.{ }^{*}, \mathrm{c}\right)$ and Qiming $\left.\mathrm{Yu}^{*}{ }^{*}, \mathrm{~d}\right)$ \\ * Electronic Engineering Institute, Hefei 230037, China. \\ a) zheng740479839@qq.com, ${ }^{\text {b) } j i n h u \_t i g e r @ s i n a . c n, ~}{ }^{c}$ vehiclefans@163.com, ${ }^{\text {d) }}$ \\ yqm1993@126.com
}

\begin{abstract}
Keywords: Data Link; TTNT Technology; Information War; Network Center Warfare.
\end{abstract}
\begin{abstract}
The essence of modern warfare is the information war, The advantage of the information is the prerequisite for the victory of modern war. With the widely used of electronic technology in the military, the struggle in electromagnetic field become more and more sharp, the information network technology has a significant impact on the update of modern warfare theory, the contrast of combat power, the changes of combat mode, the changes of battlefield environment and the process and outcome of war, it also brings epoch-making changes. Data link adds command, control, weapons, detection and other platforms together, it is neural network center of information transmission in the battle. TTNT (Tactical Targeting Network Technology system) as the latest generation of tactical data link system, is able to attack the short-term moving targets, and realize the ability of "What is discoveried will be destroyed immediately", through the using of TTNT technology, air and ground can achieve a variety of rapid networking platforms, and transfer data at a very high speed, so that it plays a decisive role in the accurate attack of time sensitive targets, and makes the army's network center warfare capabilities produce a qualitative leap. This article mainly explains the development of data link and TTNT's main technology and it's application.
\end{abstract}

\section{INTRODUCTION}

Sun Tzu said: soldiers, the large event of the country, the land of death and life , the way of exist and die, cannot but be investigated.

Since the emergence of human civilization, with the economic, political, cultural, military development, in human history, there have been all kinds of wars more than ten thousands times.in history, the production and perish of all dynasties will be launched violent war. The nature of the war is faith, nation, religion, state, alliance, interference, aggression, right, etc. The weapons used in the war from simple wood to metallized cold weapons to guns, artillery, armor, missiles, radar, satellites, and even nuclear and chemical weapons; the form of war from a single style of land, sea operations to land, sea, air, days, net, electric integration joint operations.

TTNT is an airborne wireless network communication technology which is developed to combat mobile targets. It provides high-speed, high-capacity, anti-jamming data communications for transmission delays and precision strike plans. It is one of the key safeguards for the realization of AMSTE program and AT3 technology. Tactical targeting network technology TTNT is a new network technology of battle field, it is based on the Internet Protocol (IP), supporting full-duplex communication, relating to the previous data link technology, it also has high-speed, broadband, anti-interference, large capacity and many other significant advantages.

\section{THE DEVELOPMENT OF DATA LINK TECHNOLOGY}

Tactical data link is the main data link used for radio communication between different platforms, it is used for the transmission and exchange of data information between command, weapon and other tactical data systems. In practice, the tactical data link system is generally composed of integrated platform application subsystem (intelligence acquisition subsystem, information fusion processing sub-system, command and control sub-system and task execution subsystem) and communication subsystem (information processing, transmission and communication management 
System). The following FIG.1 is the introduction of the actual using of the Link series of tactical data link system Link4, Link11, Link16, Link22 and the main technical features of the new satellite TTNT in rapid development.

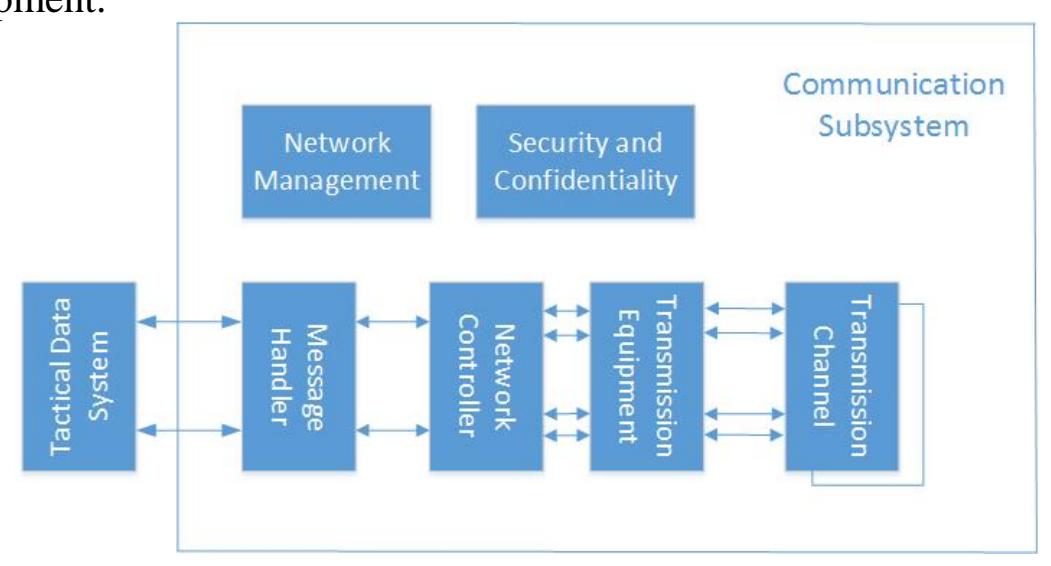

FIGURE 1.Tactical data link system block diagram.

(1) Link 4 data link

The late nineties of the 20th century, the US Navy began to develop tactical digital information chain, and gradually develop into Link4 data link, through the radio to the fighter transmission of non-confidential tactical data. It continues improving after Link4, it develops from the initial oneway transmission to supporting the development of two-way transmission of Link4A and Link4C, which can support two-way data communication between command platform and airborne platform .

(2) Link 11 data link

The United States Navy began to develop Link11from 1960s, it was put into using in 70s. The Link11 data link operates in the HF shortwave band (15to30 MHz) and the UHF ultra-short wave band (225to400 MHz) with two standard transfer rates:1.2 kb/sand2.4 kb/s. The actual using is $1.364 \mathrm{~kb} / \mathrm{s}$ or $2.25 \mathrm{~kb} / \mathrm{s}$.

(3) Link 16 data link

Link16 developed in the 1970s, compared with its predecessors, it has a good confidentiality, capacity, anti-interference, etc. using $\mathrm{J}$ sequence message format. It's operating frequency band is $960 \sim 1215 \mathrm{MHz}$, and it's transmission rate is115.2 238 kb/s, it is 100 times compared with the previous data link, using TDMA working mode and the combination of hopping spread spectrum anti-jamming mode. However, with the development of a variety of detection technologies, modern fighter (such as F-35) on the airborne small active phased array radar (AESA) in synthetic aperture mode mapping of digital map resolution has reached the order of $250 \mathrm{~mm}$, it's photoelectric sensor detection distance and infrared imaging resolution is also doubled, Link16 has been unable to meet such a huge demand for information transmission. As a result, the US military started a new data link research and development work as early as 2001, so TTNT came into being.

The development of the data link transmission rate is shown in Fig.2. As can be seen from Figure 2 , one of the basic characteristics of data link development is high transmission rate.

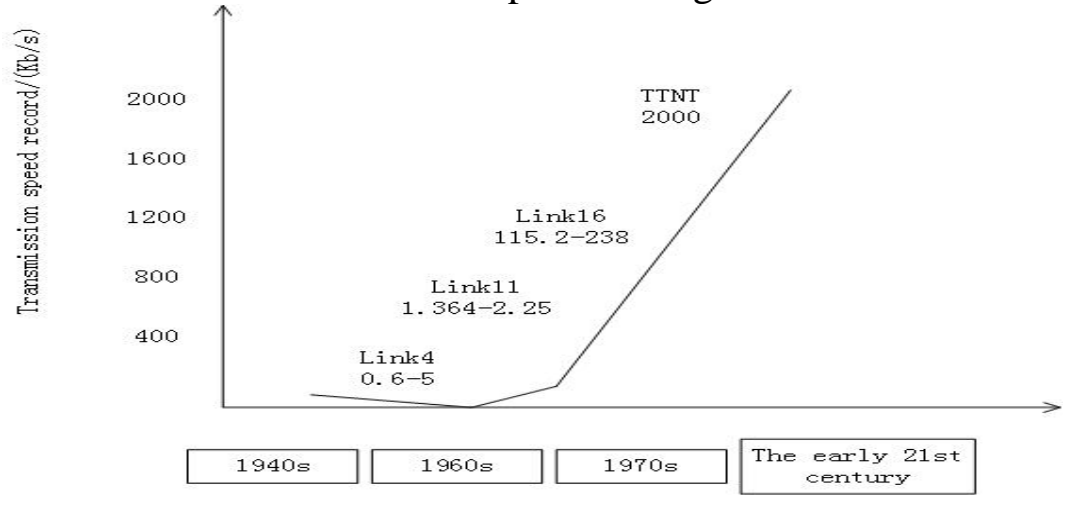

FIGURE 2. Schematic diagram of data link transmission rate. 


\section{THE ANALYSIS AND RESERCH ON TTNT DATA LINK}

\section{The Introduction of the TTNT Data Link}

Data link is a kind of military data communication system, which uses the network communication technology and application protocol to link all kinds of platforms effectively and realize the tactical information processing and transmission. It is the core and the most direct technical support force of the playing of network center warfare effectiveness, the data link plays a role of the neural network in the modern information war, it can quickly activate a variety of combat forces in full dimensional battle field, improve the combat capability of the action as "glue" and "multiplier", can effectively shorten the sensor to the shooter's reaction time, speed up the rhythm of the war. The TTNT data link is a high-speed, broadband, and new network technology of battle field based on the IP protocol, which will be embedded in the joint tactical radio system (JTRS) in the course of using. It has high throughput, high transmission rate, low transmission delay and real-time bandwidth-based performance, can make massive battlefield intelligence information and situation images and other combat platforms for high-speed transmission and realtime sharing. This will help to combat time-sensitive targets, greatly improve the ability of network center warfare. Using TTNT technology, you can achieve rapid interconnection of various platforms such as sea, land, air, sky, network, electricity and so on, and carry out data transmission at extremely high speed, so as to strike the time sensitive target accurately.

\section{The Main Technical Content of TTNT Data Link}

TTNT network is based on the IP protocol, its self-organization, distributed network structure is simple, you can quickly achieve networking .

TTNT network process as follows:

(1) Entering the network range, receiving the welcome signal. (2) Decoding the received signal and extracting the location information of the nearest node. (3) Sending authentication information to the nearest node. (4) The verification passes through, returning to register IP. Verification does not pass, continuing to require verification information. (5)Establishing the connection from the nearest node to other nodes to obtain information. (6) Uninterruptedly send their own location information to nearby nodes.

The end of the task of the aircraft to exit the network in two ways:

(1)Flying away from the network range, can not send and receive the network information. The original network can not update its location information, recovering its IP after overtime, and disconnect. (2) Manually send off the network connection request, stop sending its location information, the nearest node to receive its disconnect information, delete its IP from the connection table, and disconnect.

Main implementation technology:

(1) working frequency band

The design and input of TTNT can not interrupt and interfere with the work of Link16, it can work with Link16 at the same time. TTNT modules can be integrated in Link16's Joint Receiving and Transmitting System (JTIDS), and not add other RF units on its basis. The radius of TTNT single-node transmission should attain at least 100 nautical miles $(185.2 \mathrm{~km})$, which can be inferred from TTNT data transmission band is about $900 \sim 1500 \mathrm{MHz}$ (probably $1350 \sim 1520 \mathrm{MHz}$ ). The waveform of TTNT can be used as part of the broadband network waveform in the JTRS system. TTNT will be able to work in three bands to ensure which can build a battlefield LAN anywhere in the world, two of which may be $1350 \sim 1520 \mathrm{MHz}$ and $1750 \sim 1850 \mathrm{MHz}$.

(2) The technology of distributed channel access control

The protocol of TTNT data link channel access control adopts SPMA protocol, which has a temporary, dynamic and fast networking, distributes dynamic access, supports multi-node simultaneous communication, supports a variety of business and distinguishes between business priority QoS guarantee, even that the highest priority Business can immediately access the channel and other capabilities. The main technologies include: distributed, fast and secure network authentication algorithm; channel busy detection and prediction algorithm based on time-frequency 
analysis method; multi-channel distributed access algorithm with conflict resolution; based on channel busy and differentiated Priority multi-channel access algorithm; variable factor back-off algorithm based on load feedback and back-off window real-time updating.

(3) IP routing technology of high dynamic multi-hop

The TTNT data link routing protocol has the ability to accurately construct and maintain the network topology database, build and update the routing table in real time, and support data transmission with low delay and high reliability. The main technologies include: dynamic analysis and forecasting technology of aeronautical formation network; adaptive beacon exchange algorithm; neighbor node table construction and maintenance algorithm; real-time construction and maintenance of network topology database; formation topology control algorithm based on network topology database; real-time and reliable design of active routing protocol.

(4) The mode of antenna operating

TTNT uses a (three-dimensional) omnidirectional and directional antenna mix mode. The MAC protocol research on directional antennas shows that when the antenna is fully used, the neighbor is found to be a technical difficulty. The main problem is that when the beam is narrow, the neighboring nodes can not find each other quickly. TTNT can take omni-directional beam transmission on omni-directional channels and quickly discover neighbors, and join the network and initiate data transmission. The omni-directional channel can be independent of the traffic channel of the directional transmission data. The directional transmission of service data can reduce the probability of interception (LPI / LDI) , enhance the transmission signal anti-interference ability, and achieve more distant transmission.

(5) Rate adaptation, adaptive coding and power control

TTNT can adaptively change FEC and coding mode and power control according to the mutual distance and received signal quality, and reduce the data transmission rate automatically when the distance increases. The data transfer rate at $185 \mathrm{~km}$ distance can reach $2 \mathrm{Mb} / \mathrm{s}$; the data transfer rate at $370 \mathrm{~km}$ distance can reach $500 \mathrm{~kb} / \mathrm{s}$; the data transfer rate can reach $220 \mathrm{~kb} / \mathrm{s}$ at $555 \mathrm{~km}$ distance. TTNT directional antenna narrow beam not only can improve the system's low interception and anti-jamming, but also help to achieve high data transfer rate. In addition, TTNT is integrated into the original JTRS communication components, data encryption is also the use of Link16 original encryption system, if it want to achieve high throughput, high data rates, it has to find a way in modulation and coding, and uses a higher Order modulation mode and higher coding rate.

\section{Superiority of TTNT Data Link}

TTNT is a high-speed, broadband-based Internet Protocol (IP) based network communication system that connects air platforms to terrestrial-based global information grid (GIG) nodes. Unlike a data link that uses a dedicated communication protocol, TTNT can perform multi-layer network management and automatically adjust bandwidth to ensure that important information is sent to nodes that require it in real time and can transmit data over multiple nodes. This high-speed network uses network-centric sensor technology to interconnect data from multiple platforms, allowing sensors to transmit information on time-sensitive targets across multiple platforms, enabling precise positioning of time-sensitive targets. It is based on the Internet protocol, the use of Ad hoc technology network, this high-speed, dynamic network can make the US military can quickly aim at mobile and time sensitive targets. TTNT is used in the US Air Force's airborne network, its purpose is to achieve tactical network aimed at and accurately hit the target.

\section{Application of TTNT Technology}

The TTNT data link system will provide an idealized communication link for next-generation network targeting technology, and most of these technologies will be used to replace the Link16 data link. The Link16 data link is used to share trend-sensing data between tactical aircraft, but because of many reasons, it is not an ideal data chain for DARPA's current technology, such as Airborne Moving Surface Targeting (AMSTE) technology and advanced tactical targeting (ATTT ) Technology that uses information collected by multiple air platforms and is quickly passed between the air platforms to pinpoint and destroy moving targets. 
In addition, TTNT's low intercept rate provides a great security for stealth fighter aircraft. TTNT uses Ad hoc networks and its dynamic networking features enable US troops to quickly target mobile and time-sensitive targets. TTNT's dynamic networking features enable fighters to join the battle quickly and provide timely and effective information through TTNT in the whole process of target detection, active identification, targeting, belligerent standards, combat and confirmation destruction. For example, the F-22 Raptor's smart and situational images can be sent to the F-15 and F-16 aircraft via a terrestrial gateway for timely sharing of information. In this way, the platform can achieve large-scale, real-time joint operations, while ensuring the battlefield victory with the damage will be reduced to a minimum. In addition, TTNT will provide a network of infrastructure for the huge transformation potential of US military network-centric warfare. TTNT network diagram is depicted in Fig.3.

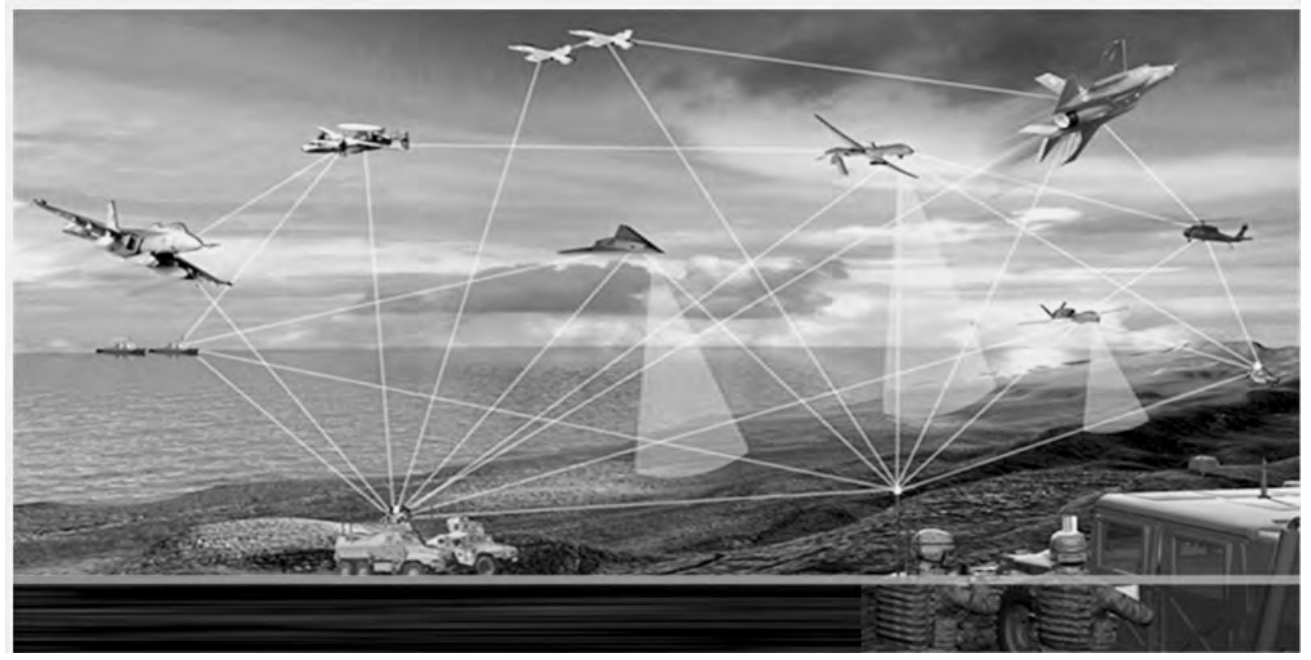

FIGURE 3. TTNT network diagram.

\section{CONCLUSION}

Through the above analysis and research on the TTNT data link, we can see that it's position in the network center war is more and more important. Throughout the development of data link, TTNT's high-speed,broadband, low latency and automatic networking features represent the future direction of the data chain. Therefore, the development of TTNT-based data link will be a key factor in future warfare. These are some technical details of TTNT on the realization of some speculation and analysis, I hope this will play a role in crack the technical details of TTNT, and these will give some inspirations to the high-speed development of military data link of our country. Of course, here's the analysis and inference, there may be some fallacies, please experts correct me.

\section{REFERENCES}

[1] Tuoyu Feng and Chunyi Liu, Data Chain Development and Network Centric Warfare, FlightMissiles, 2012.

[2] Chen Zhihui and Li Daxiang.,On the US next generation data link TTNT technologyAnalysis and discussion, communication technology, 2011.

[3] Sai Zhou, Research on Multiple Access Protocol of TTNT Data Link, Xi'an University of Electronic Science and Technology,2015.

[4] QiangYong Meng and LiXin Wang, Research on TTNT Data Link, Flying Missiles,2014.

[5] ZhiHui Chen and DaShuang Li, Analysis and Discussion on TTNT Technology of Next Generation Data Link in US Army, Communication Technology,2011.

[6] Rong Jin and HengYang Zhang, The US military TTNT Application Status of Data Chain Development, Now Navigate,2014. 
[7] JiHan Zhang, Simulation of UAV airborne communication system based on TTNT, University of Electronic Science and Technology,2013.

[8] Teerakittiul P, Tempesti G, Tyrrell A M. The applica-tion of evolvable hardware to fault robot control. IEEEWorkshop on Evolvable and Adaptive Hardware, 2009.

[9] Ting Wang, Boping Xiao, Lin Ma. An applicationinstance of FPGA in the field of PHM. IEEE Conferenceon Digital Object Identifier, 2012.

[10] Rockwell Collins. Tactical Targeting Network Technology (TTNT) [EB/OL]. http://www.rockwell-collins.com/products/gov/surface/crossplatform/comsystems/ttnt/index.html, 2008.

[11] Cruz C I. Netwars Based Study of Joint Stars Link-16 Network [D]. Wright-Patterson Air Force Base, Ohio: Air Force Inst of Tech, 2004. 